\title{
TriBot: A Minimally-Actuated Accessible Holonomic Hexapedal Locomotion Platform
}

\author{
Shadi Tasdighi Kalat, Siamak G. Faal, Ugur Celik, and Cagdas D. Onal
}

\begin{abstract}
This paper introduces a unique hexapedal locomotion platform tagged TriBot as potential agents for swarm robotic systems. We investigate the differences in performance and kinematic characteristics of two identical prototypes of this mobile robot manufactured using different design and fabrication methods and materials. Among alternatives for mass production, we focus on cut-and-assemble (CA) acrylic bodies and cut-and-fold (CF) origami-inspired polyester structures as two promising methods for manufacturing these agents. Through a comprehensive comparison between the two prototypes, advantages and disadvantages of each robot development approach are presented. This information will enable the selection of the most appropriate robotic platform according to environmental and operational task specifications.
\end{abstract}

\section{INTRODUCTION}

Swarm robotics requires a large number of cooperating agents that are capable of maneuvering in a real physical environment. Due to the large population of the group, even small improvements on fabrication time and cost of each agent will dramatically affect the accessibility of the overall system. Thus, recently, there is a growing interest in simplification of the fabrication process of potential swarm agents. To address this problem, different techniques, including: layer-by-layer 3-D printing [1], origami-like folding based fabrication [2], [3], [4], [5], [6], [7], and construction of 3$\mathrm{D}$ geometries from interlocking planar components [8] have been discussed in the literature. Layer-by-layer 3-D printing makes it possible to fabricate highly complex geometries and provides more design freedom, but the print process can be time consuming, it requires expensive facilities and tools and infiltration is required for newly printed parts [9]. As a result, 3-D printing a large population of robots is expected to be relatively inefficient.

In this article, the design process of a hexapod robot is discussed by utilizing two fabrication techniques: origamiinspired cut-and-fold structures (CF) and the assembly of 3-D structures using interlocking planar pieces, referred to as cutand-assemble (CA) throughout this article. Both techniques only require a 2-D machining device to either cut the crease patterns or cut out the interlocking pieces for the assembly process. The process of cutting a sheet of material by currently available technologies (e.g. laser cutting) is precise, fast and inexpensive [10, p. 200]. Thus, it highly impacts the time and cost of fabrication for the final products.

Cut-and-fold (CF) structures are fabricated by sequential folding of relatively thin sheets of raw material based on

Soft Robotics Laboratory, Department of Mechanical Engineering, Worcester Polytechnic Institute, 100 Institute Road, Worcester, MA 01609 \{stasdighikalat, sghorbanifaal, ucelik, cdonal\}@wpi.edu

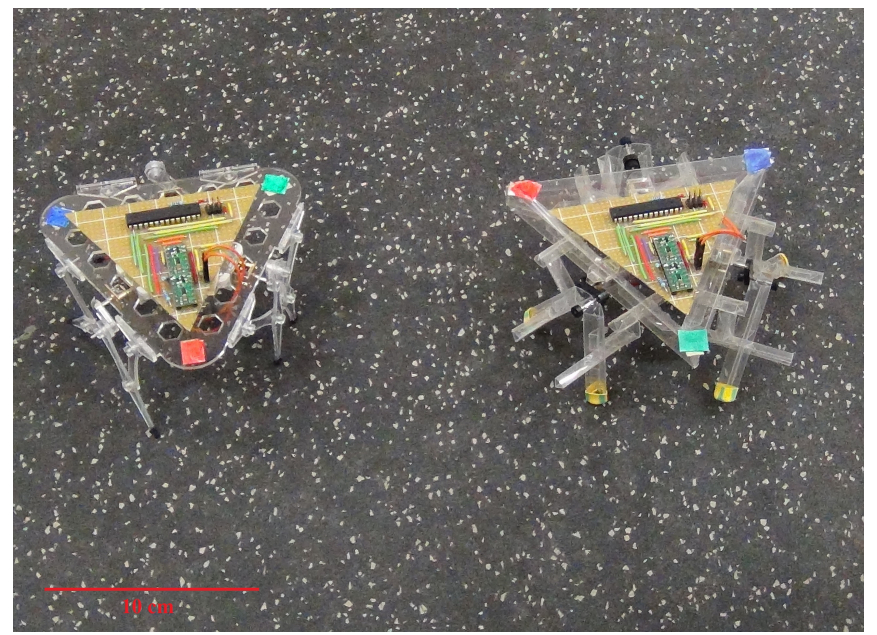

Fig. 1. Two TriBot platforms with identical designs and subsystems, manufactured using cut-and-assemble (CA) (left) and cut-and-fold (CF) (right) techniques.

predefined crease patterns as we presented in previous work [3], [4], [5], [6], [7]. Application of origami-inspired fabrication enables the construction of complicated assemblies that contain both mechanical and electrical components. As a consequence, robotic systems with fully integrated circuitry and motion transmission elements can be developed from scratch at scales and volumes not easily achievable by existing techniques. Also, the material sheets in flat form can be equipped with electrical components before assembly, which may significantly reduce the storage and shipping volume of robotic products. Thus, fabrication methods that involve folding offer a promising solution for time- and cost-effective on-demand productions. A major problem associated with $\mathrm{CF}$ techniques is the design of crease patterns. The crease patterns need to address the geometrical as well as mechanical requirements of the parts. In addition, the ability of the robots to carry meaningful payloads and withstand large reaction forces exerted on their links is highly deterministic in some applications.

Compared to origami-inspired techniques, 3-D structures can be readily manufactured by assembling planar pieces that are cut out from sheets of raw materials at a desired thickness. Thus, construction of complex 3-D structures is converted to designing and assembling interlocking parts. These parts could also be fabricated equally well using inexpensive and rapid prototyping methods (e.g. laser cutting machinery) with similar manufacturing time and cost. More- 


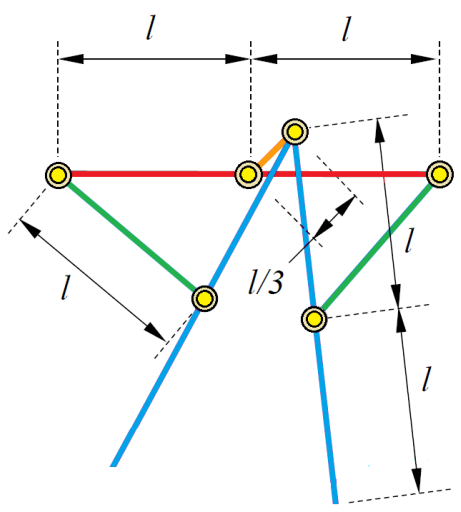

Fig. 2. The six-bar linkage mechanism used as the robot legs.

over, due to the ability to select different thickness values for individual parts, robotic platforms constructed by this method could be optimized with respect to the desired operational conditions. This feature is expected to result in significant improvements in strength and payload carrying capabilities of the final products. On the other hand, in comparison to CF structures, rigid body parts of CA robots and non-flexible joints make these mechanisms more likely to break under sudden impacts.

A wide range of tasks may be allocated to mobile swarm agents in a real-world application. To be able to perform assigned tasks, robots need to have a dexterous and agile locomotion system. Inspired by the locomotion systems of insects [11], hexapod platforms are a promising solution to take the robots out of laboratory environments. Static stability and dexterity to pass over relatively rough and uneven terrain make hexapod platforms a suitable solution for real-world applications [12], [13].

This paper presents two different approaches for construction of a novel legged hexapedal robotic platform, called TriBot, which can approximate holonomic locomotion using only three degrees of freedom (DoF). We explore the advantages and disadvantages for each platform based on experimental results. One robot is constructed by folding sheets of thin polyester by means of origami techniques, which is referred to as $\mathrm{CF}$, and the second one (CA) consists of assembled rigid parts, which were cut from an acrylic sheet. Fig. 1 presents the final prototypes with the same kinematic design, using identical electrical, mechanical, and computational components.

\section{Mechanism Design AND SYnthesis}

The TriBot is composed of three six-bar linkage mechanisms which are installed on the edges of an equilateral triangle, each one driven by a miniature rotary electric motor. This 3-DoF platform, inspired by holonomic drive locomotion, uses synchronized motion of the legs instead of wheels. As a result, by controlling the magnitude and direction of angular velocity, phase difference and geometrical position of activated actuators, various linear and rotational motions can be achieved by the robot.

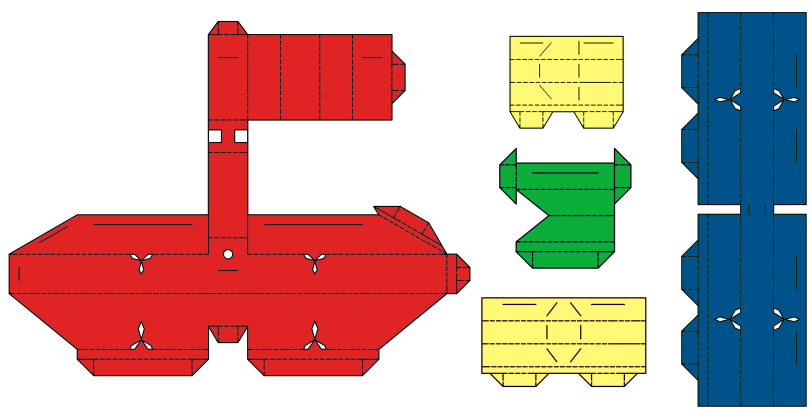

Fig. 3. Crease pattern for the cut-and-fold hexapod robot prototype.

The design of the six-bar linkages that are utilized in this platform is based on the Hoeken linkage [14]. The Hoeken linkage is a four-bar mechanism that is designed to convert crank rotation into an approximately straight-line motion. The coupler curve of the linkage resembles the motion of a foot through a gait cycle. By taking advantage of this property, it is possible to produce gait patterns of the 3DoF platform. Note that the link-length ratios of the Hoeken linkage are modified to satisfy a relatively uniform speed profile with an elliptical coupler curve (which become feet trajectory). To make the platform statically stable, this 4bar linkage is coupled with its cognate, that is 180 degree out of phase, to form a compact six-bar linkage. Note that, this is only made possible by the unique geometry of this mechanism: it is a symmetric four-bar and the coupler point is on the extended coupler link. Fig. 2 illustrates the mechanism utilized in the TriBot design. The 180 degree phase difference allows one foot to enter the stance phase while the other one is getting into the swing phase.

\section{CUT-And-Fold PlatForm}

\section{A. Design}

After designing the six-bar mechanism based on the desired trajectory of the legs, the crease pattern for folding a thin sheet of polyester was designed. Fig. 3 shows the crease pattern for the CF TriBot. The final body of this robot is formed by connecting three six-bar mechanisms shown in Fig. 4 into an equilateral triangle made by folding this crease pattern.

\section{B. Fabrication}

The CF robot is fabricated from $0.2-\mathrm{mm}$ thick polyester sheet, while any laser-machinable material can be utilized. After printing the designed crease pattern, it can be folded to form the final robot. The whole process takes 90 minutes to be completed. The CF robot is composed of five components: one equilateral triangular-shaped main body including motor holders, upper shafts, rocker links, lower shafts, and connectors that are necessary for locking upper and lower shafts to the main body for each leg. Legs are connected to each side of the main body in pairs to form the six-bar 


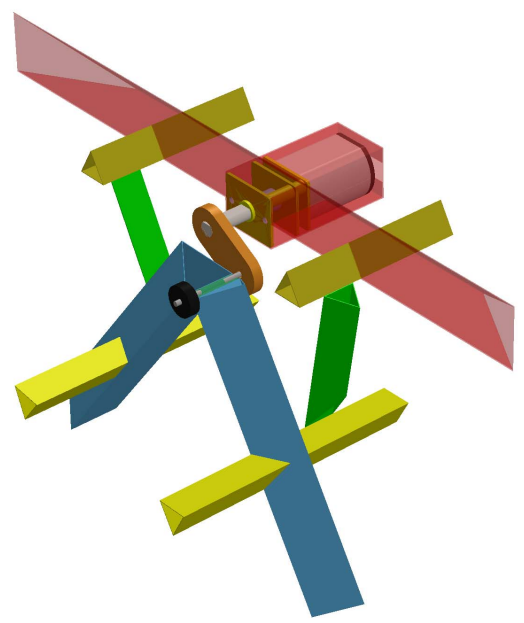

Fig. 4. Folded six-bar linkage used in the legs of the CF hexapod robot prototype.

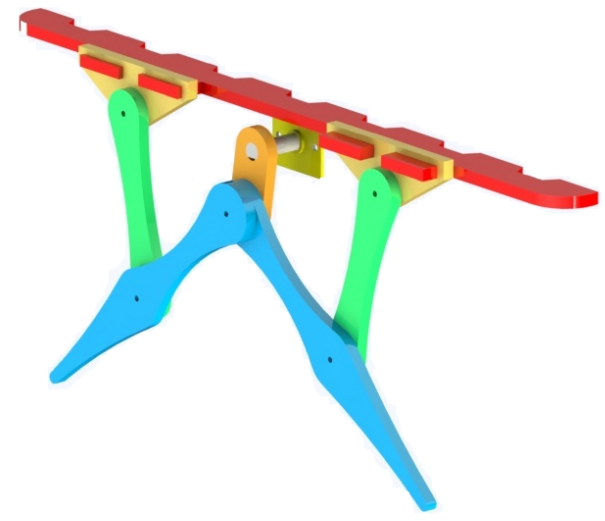

Fig. 5. Six-bar linkage assembly used in the legs of the CA hexapod robot prototype.

mechanism described above. Fig. 4 shows the final folded mechanism of the robot legs.

Embedded control circuit elements consist of Atmel Atmega81 microcontroller unit (MCU) to adjust the applied voltage to three Pololu micro gear DC motors. Pulse-widthmodulation (PWM) signals sent from the microcontroller to each motor individually drive the motors using a Pololu DC motor driver. The robot maintains its power for actuation from three single cell lithium polymer batteries $(3.7 \mathrm{~V}$, $160 \mathrm{mAh}, 4 \mathrm{~g}$ ). The total mass of the robot including the battery pack and electronic controls is 58.2 grams.

\section{Cut-And-Assemble PlatForm}

\section{A. Design}

In order to have a valid comparison between the two platforms, the CA hexapod robot platform is designed with the same dimensions as the CF counterpart. Additionally, to reduce the final mass of the robot, some material is removed from the triangular body, as depicted in Fig. 1. Different

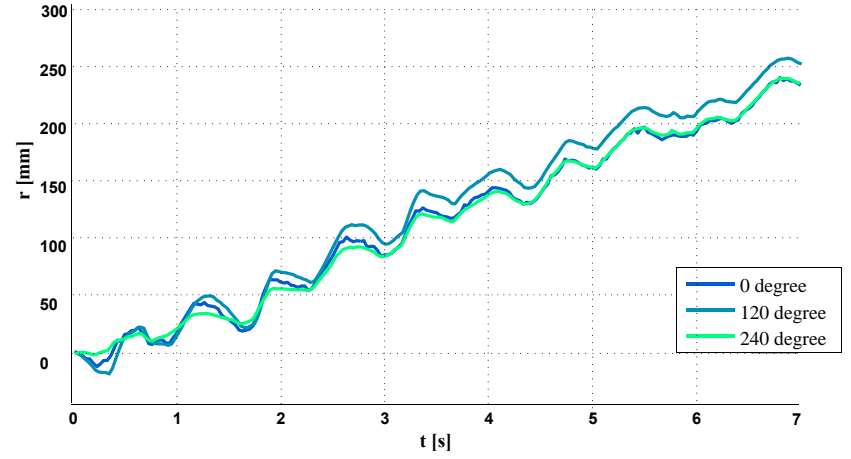

Fig. 6. Position of the center of gravity versus time in a linear motion with 120 degree increments for CA robot

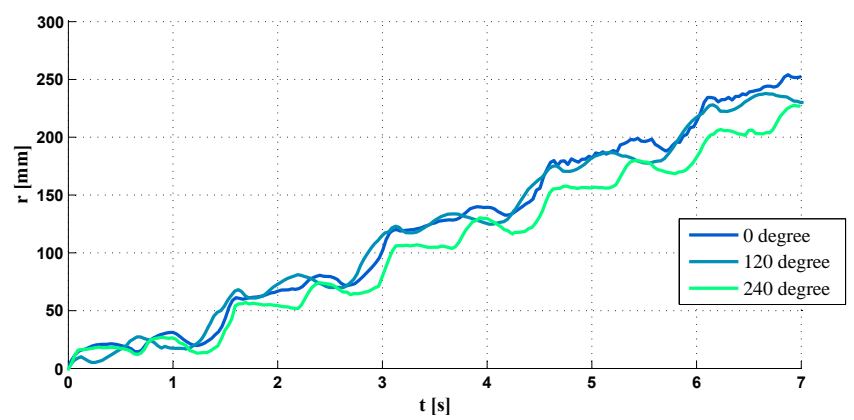

Fig. 7. Position of the center of gravity versus time in a linear motion with 120 degree increments for CF robot

parts that form one of the three six-bar-mechanism legs of the robot are illustrated in Fig. 5. Note that, for the sake of clarity, The same color coding is used in Fig. 4 and Fig. 5, corresponding to $\mathrm{CF}$ and $\mathrm{CA}$ prototypes.

\section{B. Fabrication}

Robot links were laser machined on a $2 \mathrm{~mm}$ thick acrylic sheet using a EPILOG Zing-10000 laser-cutter machine and assembled together to form the final agent. Fig. 5 shows a 3-D CAD model of the legs of the CA TriBot. The whole process for making one robot takes less than 30 minutes. As for the CF prototype, three DC motors were installed on each side of the triangular body to drive the six-bar mechanisms. The total mass of the robot including the same battery pack and control electronics is 70.3 grams.

\section{EXPERIMENTAL RESULTS}

In this section, quantitative results for comparative experiments between the two TriBot prototypes are presented and detailed explanations on each experiment is described.

\section{A. Locomotion Performance}

Performance of the platform is tested through experimenting its holonomic locomotion system. Figures 6 and 7 represent the $\mathrm{CA}$ and $\mathrm{CF}$ platforms following the desired trajectories which are defined as straight lines separated by 120 degree increments. This motion is achieved by sequentially activating two of the three six-bar linkages with the same 


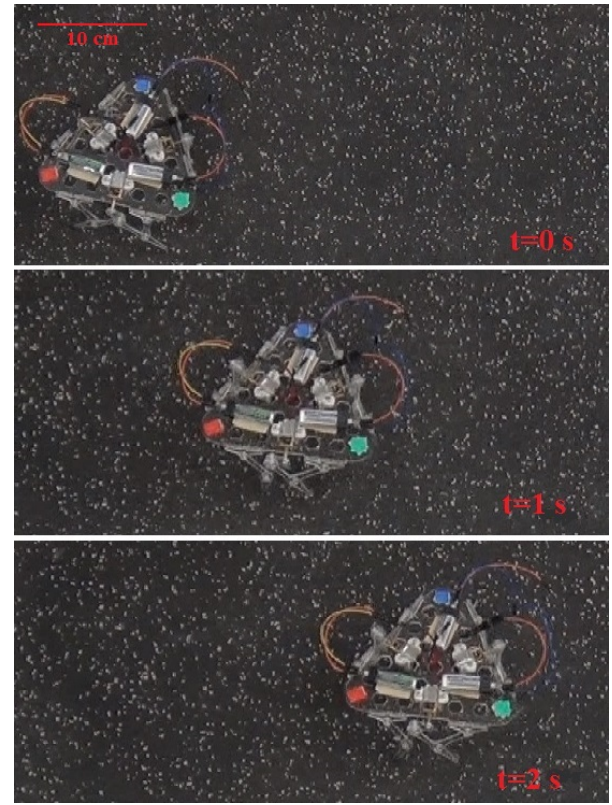

Fig. 8. Snapshots of the CA hexapod prototype undergoing linear translation.

velocity. Snapshots of representative experiments are given in Figs. 8, and 9. It is observed that, during linear translation, the phase of the inactive linkage affects the direction of motion. This effect, in combination with regulating rotational speed of the actuators and feedback control could be used in order to achieve accurate motion control of robot in any desired direction.

Figure 10 presents the trajectory of the center of gravity (CG) position, measured using color markers, for each robot while following the desired linear trajectory. In this experiment, the position of the inactive linkage is kept symmetric with respect to the triangular body plane of symmetry. As seen in the figure, the CA robot experiences less disturbances than the CF during linear motion. This difference is because of the flexible body of the CF robot making it harder to maintain a symmetrical situation.

Similarly, the TriBot platform is able to perform pure rotations by activating all three actuators in the same angular direction. Rotational performance of $\mathrm{CF}$ and CA robots was experimentally tested by measuring the angular velocity of both prototypes. Figure 11 illustrates the 3-DoF platforms rotating about an axis approximately passing trough their geometrical center. In Fig. 11, Theta represents the planar orientation of the robots. Snapshots of a representative experiment are also given in Fig. 12.

\section{B. Kinematics}

Kinematic characteristics of a robot is one of the fundamental decision factors in selecting an appropriate robotic locomotion platform for task specifications. Due to the inherent flexibility of CF links and joints, they may experience changes in shapes and dimensions under load, which lead to variations in kinematic properties for them. To demonstrate
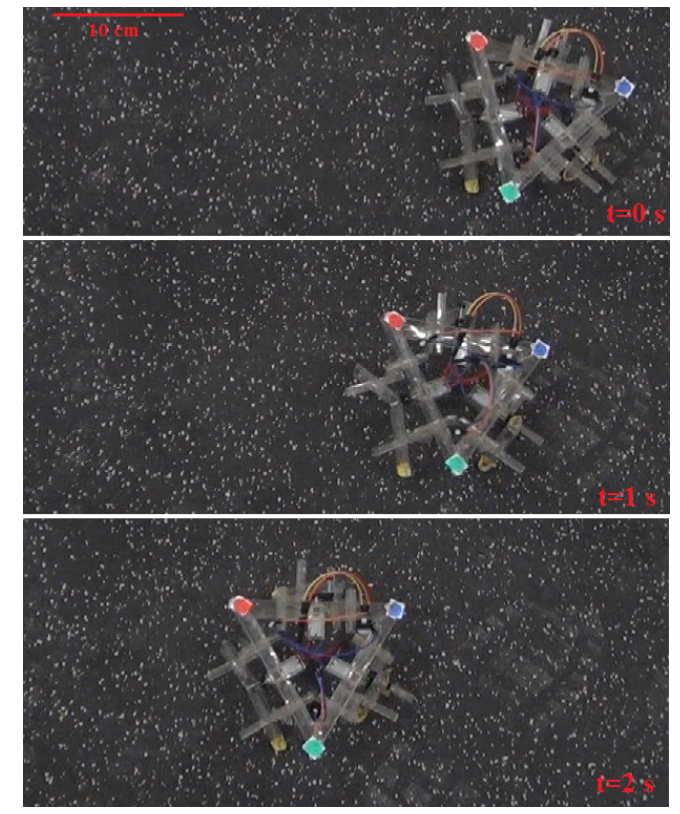

Fig. 9. Snapshots of the CF hexapod prototype undergoing linear translation.

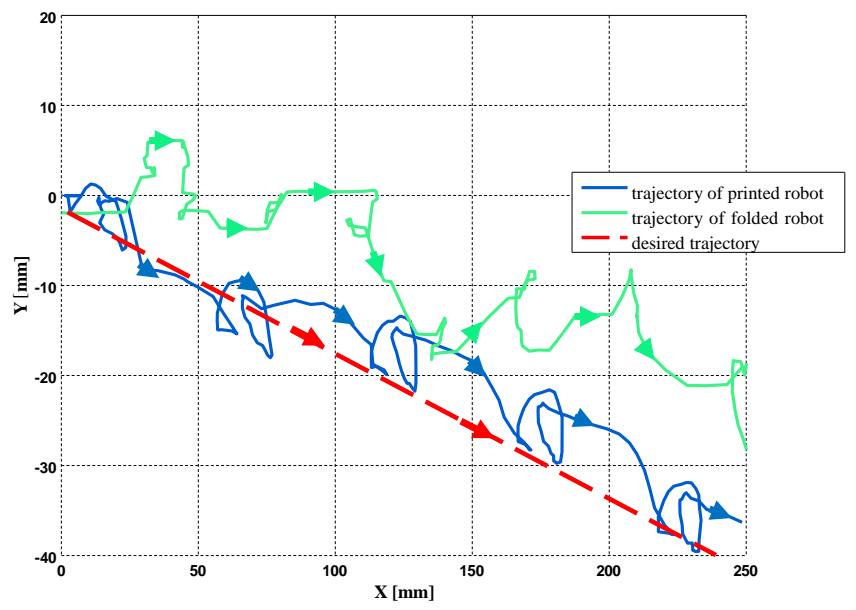

Fig. 10. CG trajectory of two robots following the desired trajectory defined as a straight line

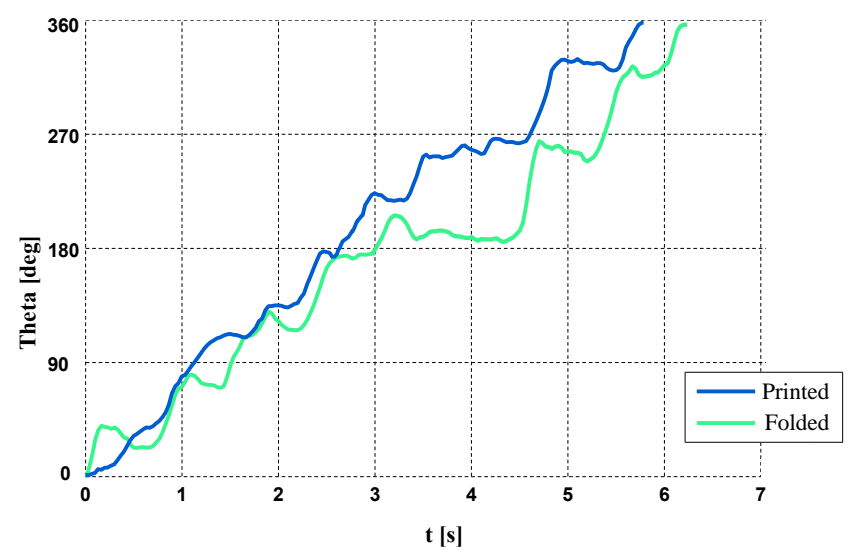

Fig. 11. Changes in the robot orientation over time 

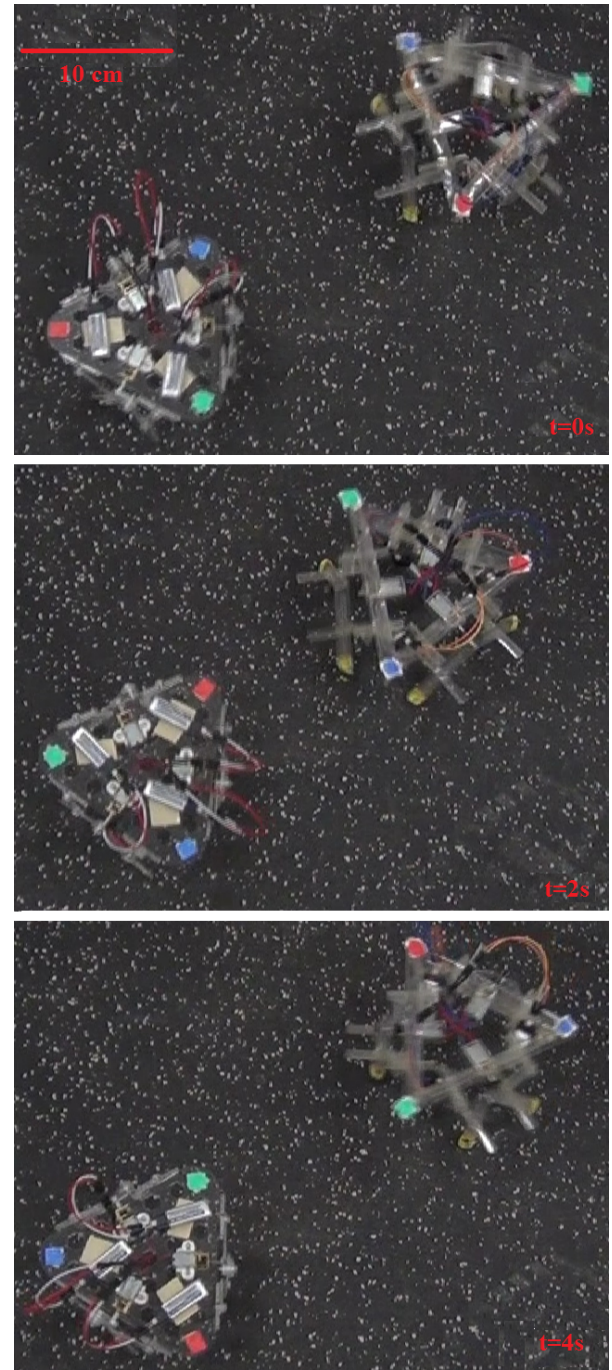

Fig. 12. Snapshots of the both prototypes undergoing pure rotation around their approximate geometric center.

the effects of this embedded flexibility, kinematic characteristics of both robots were measured experimentally.

The step length for each robot was obtained by placing a marker at the end of the coupler link (at the foot) and analyzing its motion as shown in Figs. 13, 14. It could be observed from these figures that both TriBots have the same step-length, when no external load is applied on their links.

\section{Mechanism Performance under Loading}

The performance of the legs of both TriBots is tested experimentally under different loading conditions. The experimental setup is composed of the 3-DoF platform and a motion capture system to record the trajectory of the tested foot (coupler curve). To conduct the experiment, the body of the robot is secured to a rigid surface with the legs pointing upward. Different weights of 20, 40 and $60 \mathrm{~g}$ are mounted on one of the feet to replicate the effect of the forces exerted to one of the legs during robot locomotion. Based on the total mass of the robot and the fact that the total weight of the robot is supported by at least three legs, the weights

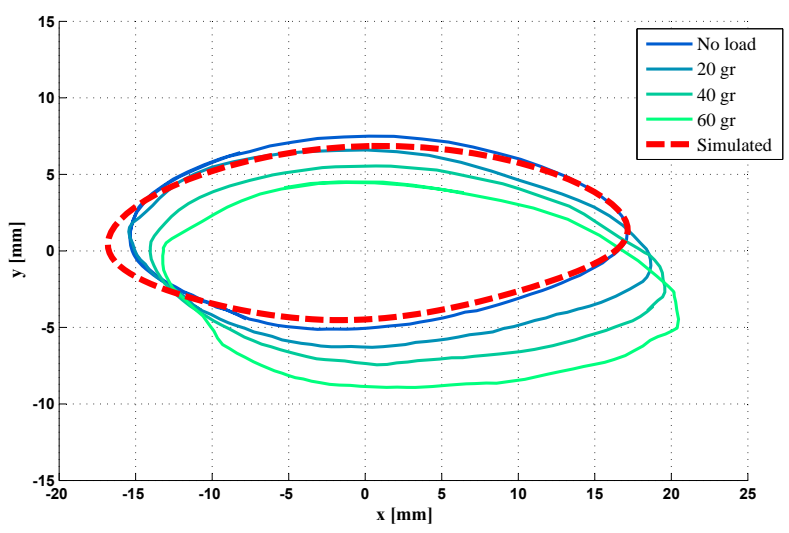

Fig. 13. Changes in the coupler curve of the CF TriBot prototype under different loading conditions.

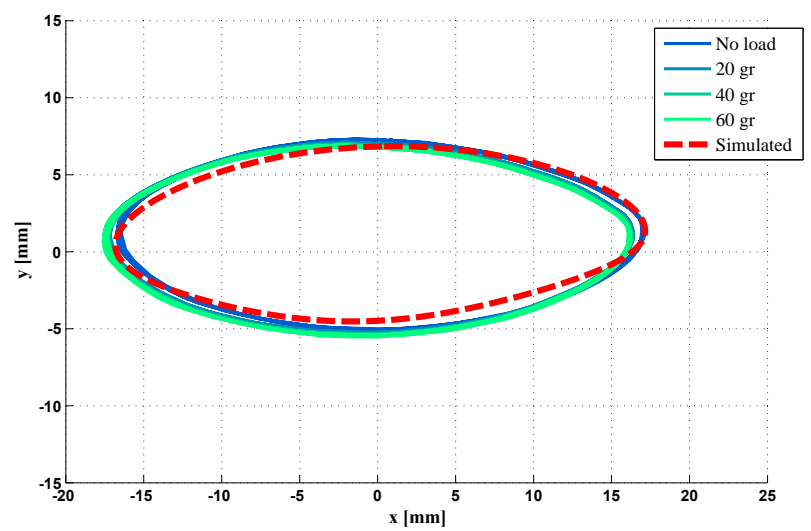

Fig. 14. Changes in the coupler curve of the CA TriBot prototype under different loading conditions.

approximately represent total payloads of 1,2 , and 3 bodyweight loading.

Figure 13 illustrates the results of this experiment for the CF TriBot. As observed in this figure, the mechanism approximately reproduces the expected coupler curve under no external loading. As the amount of payload increases, the coupler curves shift toward positive $\mathrm{x}$ axis and negative $y$ axis. The shifting effects are due to the moments and torsions exerted on the triangular beam of the base of the robot. It is also observed that increasing the payload value, introduces some distortions on the coupler curve when crank angle approaches 180 degrees due to the slot used to connect the crank to the coupler link.

In contrast, as shown in Fig. 14, applying external load on CA robot mechanism slightly shifts the desired coupler curve in the first step. this might be due to small gaps in mechanism joints. As a result of rigid links, despite increasing the amount of load on the leg to greater values, the coupler curve remains almost identical to the expected shape.

\section{Power consumption}

The power consumption rate for both TriBots is measured by the continuous operating period (lifetime) with one battery 
charge. in this experiment, no external loading was applied on the robots. Each battery pack consisted of three one-cell lithium polymer batteries $(3.7 \mathrm{~V}, 160 \mathrm{mAh})$. As expected, the CA robot has a slightly higher rate of power consumption due to its larger mass. The maximum continuous operation time for the CF TriBot is 247 minutes, while it was 225 minutes for the CA TriBot.

\section{E. Maximum payload}

The maximum payload the TriBots can carry was measured by the process of incrementally increasing the amount of payload on their main body. The payload was distributed in a triangular form and consisted of connected $20 \mathrm{~g}$ weights. A current limitation of $1.7 \mathrm{~A}$ was applied to the power supply in order to prevent any damage to the electric motors. The maximum amount of carried payload by the CA robot is $1322 \mathrm{~g}$ and for the $\mathrm{CF}$ robot, $526 \mathrm{~g}$. It is remarkable that the experiment with the $\mathrm{CA}$ robot had to be terminated when the power supply hit the 1.7-A current limit and not because of structural problems.

TABLE I. Performance characteristics of the two TriBot hexapedal locomotion prototypes.

\begin{tabular}{l||c|c}
\hline Agent & CA & CF \\
\hline \hline Total Mass $(\mathrm{g})$ & 70.3 & 58.2 \\
\hline Maximum Payload $(\mathrm{g})$ & 1322 & 526 \\
\hline Cost of Transport & 22.8 & 20.20 \\
\hline Step length $(\mathrm{mm})$ & 3.6 & 3.6 \\
\hline VAverage at $0^{\circ}(\mathrm{mm} / \mathrm{s})$ & 36.43 & 36.28 \\
\hline VAverage at $120^{\circ}(\mathrm{mm} / \mathrm{s})$ & 34.28 & 32.86 \\
\hline VAverage at $240^{\circ}(\mathrm{mm} / \mathrm{s})$ & 34.43 & 32.43 \\
\hline VAngular $(\mathrm{rev} / \mathrm{min})$ & 10.52 & 9.67 \\
\hline Fabrication Time $(\mathrm{min})$ & 30 & 90 \\
\hline Cost of Fabrication $(\mathrm{USD})^{*}$ & $1.32+C^{* *}$ & $1.14+C$ \\
\hline & $*$ The cost of platform fabrication is calculated on July \\
29, 2015. & ** C is the cost of actuators and electronics that is the \\
same for both platforms which is approximately equal \\
to 10 USD.
\end{tabular}

\section{CONCLUSION}

A summary of all the measured characteristics of the two TriBot platforms is presented in Table. I. As the experimental data indicate, both platforms demonstrate a very similar kinematic behavior that includes similar linear and angular velocities and length of steps. However, due to the rigidity of the links, the CA prototype moves more uniformly and experiences less disturbances and deviations as it follows a defined open-loop trajectory. On the other hand, the $\mathrm{CF}$ prototype weighs less than the CA counterpart; thus it has a lower cost of transport.

The maximum allowable payload of the CA platform is more than 2.5 times the $\mathrm{CF}$ platform, while the payloadto-mass ratio of the $\mathrm{CA}$ platform is more than two times larger than the $\mathrm{CF}$ platform (18.8 vs 9). Moreover, the links of the CA platform show less deflection under loading. This behavior is established in Figs. 13 and 14.

The relative rigidity and high payload carrying capacity of the CA fabrication technique makes it a suitable choice to fabricate platforms which require precision and structural stiffness. On the other hand, the CF based fabrication results in lower weight for the structure and shows an inherent flexibility. These features are advantageous for applications that require lower transportation energy and higher compliance.

Between the two TriBot platforms, the CA platform is an ideal alternative for applications that require object manipulation or material handling, while the lighter weight and lower cost of transport of the CF TriBot makes it a good choice for applications that require longer operation time. Although it has not been tested definitively in this paper, the inherent flexibility of the CF Tribot is expected to be advantageous for maneuvering on uneven terrain [12].

Throughout this study, the performance of the platforms are measured based on their ability to follow open-loop control commands. Thus, applying closed-loop feedback control on joint positions to increase the accuracy of the steps will be one of the future research directions stemming from this work.

\section{ACKNOWLEDGMENTS}

The authors would like to thank Fuchen Chen, Erik H. Skorina, and Saber Jahanpour for their generous help on this manuscript.

\section{REFERENCES}

[1] H. Lipson and M. Kurman, Fabricated: The new world of 3D printing. John Wiley \& Sons, 2013.

[2] A. M. Hoover and R. S. Fearing, "Fast scale prototyping for folded millirobots," in Robotics and Automation, 2008. ICRA 2008. IEEE International Conference on, pp. 886-892, IEEE, 2008.

[3] C. D. Onal, R. J. Wood, and D. Rus, "Towards printable robotics: Origami-inspired planar fabrication of three-dimensional mechanisms," in Robotics and Automation (ICRA), 2011 IEEE International Conference on, pp. 4608-4613, 2011.

[4] C. D. Onal, R. J. Wood, and D. Rus, "An origami-inspired approach to worm robots," IEEE Transactions on Mechatronics, pp. 430-438, 2012.

[5] M. Agheli, S. G. Faal, F. Chen, H. Gong, and C. D. Onal, "Design and fabrication of a foldable hexapod robot towards experimental swarm applications," in IEEE International Conference on Robotics and Automation (ICRA), 2014.

[6] S. G. Faal, F. Chen, W. Tao, M. Agheli, S. Tasdighikalat, and C. D. Onal, "Hierarchical kinematic design of foldable hexapedal locomotion platforms," JMR, vol. 3, no. 4L3, p. 1, 2015.

[7] C. D. Onal, M. T. Tolley, R. J. Wood, and D. Rus, "Origami-inspired printed robots," IEEE Transactions on Mechatronics, 2015.

[8] Y. Schwartzburg and M. Pauly, "Fabrication-aware design with intersecting planar pieces," in Computer Graphics Forum, vol. 32, pp. 317326, Wiley Online Library, 2013.

[9] S. Upcraft and R. Fletcher, "The rapid prototyping technologies," Assembly Automation, vol. 23, no. 4, pp. 318-330, 2003.

[10] N. B. Dahotre and S. Harimkar, Laser fabrication and machining of materials. Springer Science \& Business Media, 2008.

[11] A. T. Baisch, P. Sreetharan, and R. J. Wood, "Biologically-inspired locomotion of a $2 \mathrm{~g}$ hexapod robot," in Intelligent Robots and Systems (IROS), 2010 IEEE/RSJ International Conference on, pp. 5360-5365, IEEE, 2010.

[12] P. Birkmeyer, K. Peterson, and R. S. Fearing, "Dash: A dynamic $16 \mathrm{~g}$ hexapedal robot," in Intelligent Robots and Systems, 2009. IROS 2009. IEEE/RSJ International Conference on, pp. 2683-2689, IEEE, 2009.

[13] D. E. Soltero, B. J. Julian, C. D. Onal, and D. Rus, "A lightweight modular 12-dof print-and-fold hexapod," in IEEE/RSJ International Conference on Intelligent Robots and Systems, 2013.

[14] R. L. Norton, Design of machinery: an introduction to the synthesis and analysis of mechanisms and machines. McGraw-Hill Professional, 2004. 